\title{
Expression Profiles and Prognostic Values of BOLA Family members in Ovarian Cancer
}

\author{
Mingyang Zhu \\ Shengjing Hospital of China Medical University \\ Shiqi Xiao ( $\sim$ aa18940258765@163.com) \\ Shengjing Hospital of China Medical University https://orcid.org/0000-0001-5684-6580
}

\section{Primary research}

Keywords: BOLA Family members, ovarian cancer, prognosis, database, bioinformatics analysis

Posted Date: October 23rd, 2020

DOI: https://doi.org/10.21203/rs.3.rs-95044/v1

License: (c) (1) This work is licensed under a Creative Commons Attribution 4.0 International License. Read Full License 


\section{Abstract}

Background: The BOLA gene family, comprising 3 members, is mainly involved in the regulation of intracellular iron homeostasis. Emerging evidence suggests that BOLA family member 2 play vital roles in tumorigenesis and progression of hepatic cellular carcinoma. However, little known about its roles in ovarian cancer.

Methods: In present study, we investigated the expression profiles, prognostic roles, and genetic alterations of three BOLA family members in patients with ovarian cancer through several public databases, containing Oncomine and Gene Expression Profiling Interactive Analysis, Human Protein Atlas, KaplanMeier plotter and cBioPortal. Then, we constructed the protein-protein interaction networks of BOLA proteins and their interactors by using String database and Cytoscape software. In addition, we performed the Gene Ontology and Kyoto Encyclopedia of Genes and Genomes pathway enrichment by the Annotation, Visualization, and Integrated Discovery database. Finally, we explored the mechanisms underlying the involvement of BOLA family members in $\mathrm{OC}$ by using gene set enrichment analysis.

Results: The mRNA and protein expression levels of BOLA2 and BOLA3 were heavily higher in ovarian cancer tissues than that in normal ovarian tissues. Dysregulated mRNA expressions of three BOLA family members were significantly associated with prognosis in overall or subgroup analysis. Moreover, genetic alterations also occurred in three BOLA family members in ovarian cancer. Network analysis and enrichment analysis indicated that three BOLA family members and their 20 interactors were mainly associated with metal-ion binding and protein disulfide oxidoreductase activity. Gene set enrichment analysis indicated that BOLA family members were mainly associated with oxidative phosphorylation, proteasome, protein export and glutathione metabolism in ovarian cancer.

Conclusions: In brief, the present comprehensive bioinformatics analysis revealed that BOLA1, 2, and 3 may be new prognostic biomarkers, and BOLA2 and BOLA3 may be a potential therapeutic target of precision therapy for patients with ovarian cancer, but further studies are demanded to certify this notion.

\section{Introduction}

Ovarian cancer (OC) is a gynecological malignant tumor ranking fifth leading cause of cancer-related death in women [1]. Approximately 295,414 cases were new diagnosed and 184,799 cases died in 2018 [2]. Most patients are diagnosed at an advanced stage due to lacking of obvious symptoms of OC in early stage and inadequate predictive biomarkers [3], leading to an extremely low 5-year survival rate [4]. In developed countries, despite diagnosis and treatment has developed recently, the survival rates of $\mathrm{OC}$ improved little for decades. Therefore, to explore gene signatures related to $\mathrm{OC}$ progression and prognosis and identification of new biomarkers for predicting prognosis and directing treatment strategies of OC is a crucial clinical challenge of important significance.

The BOLA gene family, a kind of non-classical secreted proteins, contains BOLA family member 1 (BOLA1), BOLA2 and BOLA3, which is widely conserved crosswise Gram-negative bacteria and eukaryotes [5]. Previous studies showed BOLA proteins were mainly linked to stress response, iron homeostasis and iron-sulfur cluster assembly and trafficking [6]. Recent studies showed BOLA2 played key roles in biology and prognosis of hepatic cellular carcinoma(HCC) $[7,8]$. However, little is known about the function of the BOLA gene family in other cancers.

In present study, we analyzed the expression status of different BOLA family members and their prognostic values to comprehensively evaluate the roles of BOLA family in OC based on different online databases, which intended to elucidate the clinical value of various BOLA family members to provide potential prognostic biomarkers and new individualized targets for OC.

\section{Materials And Methods}

Oncomine database analysis for the expression patterns of BOLA members in OC. The differential expression of BOLA members between normal controls and OC samples was performed by Oncomine database (www.oncomine.org), an online cancer microarray database and web-based data-mining platform [9]. The search contents and thresholds were set as follows: keywords, BOLA1, BOLA2 and BOLA3, primary filter, cancer vs. normal; cancer type, OC, sample type, clinical specimen; data type, mRNA; the absolute value of fold change $>1.5, P<0.05$; and gene rank, $10 \%$. Using the Student's $t$ test to calculate the $P$ value.

\section{Gepia And Hpa Database Analysis For The Validation}

The Gene Expression Profiling Interactive Analysis (GEPIA) database (http://gepia.cancer-pku.cn/), a new web-based tool, supplies online analysis of data from The Cancer Genome Atlas (TCGA)[10]. In our study, we used it to perform the differential mRNA expression analysis of BOLA family genes between OC samples and normal controls, differential mRNA expression analysis of BOLA family genes in different pathological stages, and correlation analysis between the expression BOLA family genes. The Human Protein Atlas (https://www.proteinatlas.org/) offers great amounts of transcriptomics, proteomics data and IHC-based protein expression patterns in specific normal human tissues compared with tumor tissues[11]. In the present study, we utilized it to systematically screen the available immunohistochemistry images of BOLA family proteins presented in the database, and then typical images were selected to show the different expression in normal ovarian tissues and OC tissues.

The Kaplan-Meier Plotter analysis of the prognostic value of BOLAs in OC 
The Kaplan-Meier plotter (http://kmplot.com/analysis), which is an online database that can be used to evaluate the values of 54,675 genes in survival rates of ovarian[12], breast [13], lung [14]and gastric cancer patients [15]. In present study, we analyze the prognostic significance of BOLA family genes in OC patients by it, the selected OC samples were split into two groups based on auto selected best cutoff. Three BOLA family members (BOLA1, BOLA2 and BOLA3) were put into the database to acquire Kaplan-Meier survival plots, meanwhile the subgroup survival analysis according to histology, grade, stage, TP53 mutation status, applied chemotherapy were performed. The hazard ratio (HR) with 95\% confidence intervals (Cls) and log-rank P values were calculated and listed in survival plots. $P<0.05$ was considered statistically significant.

\section{cBioPortal database analysis of genomic alteration of BOLAs in OC}

The cBioPortal for Cancer Genomics (http://cbioportal.org), an open-access web resource,offers multidimensional cancer genomic data from TCGA [16]. In the present study, one TCGA dataset of OC named TCGA Provisional (606 cases) was selected to be further analyzed for BOLAs gene mutations or copy number alterations (CNA) in OC. The oncoPrint, co-expression, mutations and survival tabs were applied according to the online instructions of the cBioPortal.

\section{GO and PPI analysis for function and interaction of BOLAs in OC}

The gene-gene interaction of BOLA family gene and their interactors network was built via the Gene Multiple Association Network Integration Algorithm (GeneMANIA; https://www.genemania.org) [17] and the Search Tool for the Retrieval of Interacting Genes Database (STRING v.10.0; https://string-db.org/) [18] was used to set up a protein-protein interaction (PPI) network. The Cytoscape software was utilized to conceive network graphs for PPI analysis [19].Enrichment analysis of Gene Ontology (GO)and Kyoto Encyclopedia of Genes and Genomes (KEGG) pathway of BOLA family genes and their interactors were explored via the Database for Annotation, Visualization and Integrated Discovery (DAVID; v.6.8;https://david.ncifcrf.gov/home.jsp) [20].

\section{Gene Set Enrichment Analysis (gsea)}

To explore the possible mechanism associated with the involvement of BOLA members in the carcinogenesis of OC, we performed GSEA by GESA program from sangerbox software (http://sangerbox.com/) [21] to find out the pathways connected to the diverse BOLA expression in the TCGA OC tissues. The annotated gene set file c2.cp.kegg.v6.0.symbols.gmt (from the MSig database) was used for reference. NES (normalized enrichment score) >+/-2, NOM Pval (nominal $P$ value) $<0.05$ and FDR (false discovery rate) $<0.05$ were evaluated for statistical significance.

\section{Results}

\section{Expression profiles of BOLA family members in OC}

We firstly analyzed dysregulated transcriptional levels of BOLA family members in 20 various types of human cancers in the Oncomine database. As listed in Fig. 1, BOLA family members might act as either tumor promoter or suppressor in diverse kinds of tumors. In overall OC patients, the mRNA expression levels of BOLA1 and BOLA2 were significantly upregulated than that in normal ovarian tissues in Bonome's dataset with a fold change of 1.373 and 3.545 respectively, however, no data on the differential mRNA expression of BOLA3 in overall OC tissues compared with normal ovarian tissues, as shown in Table 1. We also summarized the mRNA levels of BOLA family members in different types of OC that obtained from Oncomine datasets in Table I. For BOLA1, the mRNA expression level was significantly higher in serous, endometrioid, mucinous, and clear cell adenocarcinoma than normal ovarian tissues in Lu's dataset. With regard to BOLA2, the mRNA expressions were higher in serous, endometrioid and clear cell adenocarcinoma in Lu's dataset and were higher in serous, endometrioid, mucinous and clear cell adenocarcinoma than normal ovarian tissues in Hendrix's dataset. As to BOLA3, the mRNA expressions were higher in serous, endometrioid, and mucinous adenocarcinoma than that in normal ovarian tissues.

Additionally, the GEPIA database was utilized to contrast the differential mRNA expression of BOLA family members between OC and normal ovarian tissues as well. As shown in Fig. 2A, the expression levels of BOLA2 and BOLA3 were remarkably higher, and mRNA level of BOLA1 was slightly upregulated $(P>0.05)$ in OC tissues than normal ovarian tissues, which corresponded with results of the Oncomine database except for that of BOLA1. Moreover, the correlation between mRNA expression levels of BOLA family members and different stages of OC were also analyzed, and only BOLA3 was significantly upregulated in higher stage (Fig. 2B).

Moreover, we analyzed the protein expression of BOLA family members in normal ovarian tissues and OC tissues using HPA database. As shown in Fig. 3, we found that ovarian stroma cells had medium BOLA1 staining in 3 cases of normal ovarian tissues. Relatively, among 11 cases of OC tissues examined, 2 cases had medium BOLA2 staining, 5 cases had low BOLA2 staining and 4 cases had no BOLA1 staining. As to for BOLA2, it was undetected in normal ovarian tissues,however, among the examined 12 OC tissues, 9 cases had medium BOLA2 staining, 1 case had low BOLA2 staining and 2 cases had no BOLA2 staining. Considering BOLA3, the data showed that there was no BOLA3 staining in normal ovarian tissues $\square$ in comparison, among 11 cases of OC tissues examined, there were 2 cases of mediumBOLA3 staining, 3 cases of low BOLA3 staining and 6 cases of no BOLA3 staining.

Taken together, these results indicated BOLA family members may function as oncogenes in OC, and may be a possible therapeutic target of precision therapy for patients with OC.

Prognostic value of BOLA family members in patients with OC 
We firstly appraised the relationship between the mRNA expression of BOLA family members and the survival in all OC patients via Kaplan-Meier plotter analysis. The data demonstrated that the increased BOLA3 mRNA level was associated with shorter PFS and OS of OC patients, while decreased BOLA2 mRNA level was associated with shorter PFS of OC patients (Fig. 4). However, there was no relation between BOLA3 mRNA level and prognosis of OC patients.

Then, we also assessed the prognostic values of BOLA family members in different subtypes of OC patients who were defined according to different histology, clinical stages, pathological grades, and TP53 status by Kaplan-Meier plotter analysis. As shown in Table II. As to OS, increased mRNA expression of BOLA3 was significantly related to shorter OS in serous OC patients for different histology. For clinical stages, low mRNA expression of BOLA2 predicted poor OS in stage 2, low mRNA expression of BOLA3 was related to shorter OS in stage 4, while high mRNA expression of BOLA3 predicted the poor OS in stage 3. In terms of pathological grades, high BOLA1 mRNA expression was linked to poor OS in grade 1-2, while high mRNA expression of BOLA2 and BOLA3 predicted favorable OS in grade 3. Interestingly, increased expression of BOLA2 predicted favorable OS in mutated TP53 type, while increased expression of BOLA1 was associated with longer OS in wild-type TP53.

As referred to PFS (Table III), increased mRNA expression of BOLA3 were significantly related to shorter PFS in serous OC patients, and decreased levels of BOLA2 predicted poor PFS in serous OC patients. For clinical stages, high mRNA expression of BOLA2 were found to be connected to longer PFS in stage 1 , stage 2, stage 3 and stage 4, high mRNA expression of BOLA1 predicted longer PFS in stage 3 , whereas low levels of BOLA1 predicted longer PFS in stage 4. For pathological grades, increased levels of BOLA2 predicted better PFS in all grades, high mRNA expression of BOLA1 and BOLA2 were remarkably related to shorter PFS in grade 1-2, while low expression of BOLA3 predicted shorter PFS. Moreover, increased expression of BOLA2 was correlated with poor PFS in OC patients either with mutated TP53 or wild type.

In general, these results suggested that the mRNA expression levels of BOLA family members could be considered as optional biomarkers for predicting the survival of $O C$ patient.

\section{Genomic Alteration Of Bola Family Genes In Oc}

We then explored the possible mechanisms that involved in dysregulation of BOLA family members' expression in OC. We analyzed the genetic alteration frequency of BOLA family members using the cBioPortal online tool for OC (The Cancer Genome Atlas, Provisional), 606 patients were analyzed totally. The oncoprints included missense mutation, deletion, and amplification, with the ratios of genetic alterations of BOLA family members in OC varied from 1.899.95\% (Figs. 5A, B). The ratios of genetic mutation in BOLA1, BOLA2, BOLA3 were $9.95 \%$ (0.17\% mutation, $9.43 \%$ amplification, 0.34 deep deletion), $1.89 \%$ (amplification), $2.06 \%$ (1.89\%amplification, $0.17 \%$ mutation) respectively. In addition, missense mutation was identified in BOLA1(Figs. 5C). Moreover, the survival curves indicated that cases with or without alterations in one of the BOLAs was not related with OS and PFS (Figs. 5C, D) using Kaplan-Meier plot analysis and log-rank test.

\section{Function And Interaction Of Bola Family Members}

A network of three BOLA family members and 20 kinds of proteins that were associated with BOLA family members significantly was set up using the String database and Cytoscape software. The network graph showed that metal-ion binding-related genes including glutaredoxin 5 (GLRX5), glutaredoxin 3 (GLRX3), werner helicase interacting protein 1 (WRNIP1), Zinc finger protein 576 (ZNF576), thimetoligopeptidase(THOP1), fumarylacetoacetate hydrolase domain-containing protein 2A(FAHD2A) and iron-sulfur cluster co-chaperone protein HscB (HSCB) were associated with BOLA family members(Fig. 6A). Next, GO enrichment and KEGG pathway analysis of BOLA family members and their interactors were conducted using DAVID. The BOLA family members were mainly related to mitochondrion and mitochondrion matrix location, BOLA family members and their interactors exerted their functions primarily on metal ion binding and protein disulfide oxidoreductase activity (Fig. 6B). However, no KEGG pathway for BOLA family members and their interactors was enriched. Finally, the Pearson correlation coefficients were calculated between BOLA family members by using correlation analysis in GEPIA and cBioPortal databases, ranging from 0.21 to 0.56 (Fig. 6C).

\section{Gsea Identifies Bolas-regulated Pathways In Oc}

To investigate the alteration of BOLA-related pathways in OC, GSEA analysis in OC with high or low expression levels of each BOLA gene were performed, gene sets with a Normalized Enrichment Score (21) $>+/-2$, FDR $<0.05$ and $p<0.05$ were identified as the hallmark gene sets, as shown is Fig. 7 . In the pathway enrichment analysis, a high expression of BOLA1 were positively correlated with several oxidative phosphorylation (Fig. 7A), while negatively correlated with the focal adhesion in OC (Fig. 7B). High expression of BOLA3 expression was positively correlated with oxidative phosphorylation, proteasome, protein export and glutathione metabolism in OC (Fig. 7C, 7D, 7E, 7F). However, there was no any hallmark gene sets enrichment for high or low BOLA2 expression in OC.

\section{Discussion}

Till now, prognostic of patients with OC remains poor and could be attributed to the lack of valuable biomarkers for early diagnosis, prognosis evaluation, and precision therapy. In hence, it is vital to learn about the gene signatures associated with the genesis and development of OC to pick out new molecular markers for early diagnosis, target therapy and evaluating prognosis. Former studies have reported that BOLA family members' functions as vital regulatory factors for intracellular iron homeostasis in the micro-environment [6, 22]. More and more evidence has suggested that iron homeostasis dysregulation is

Page 4/15 
connected to oncogenesis and development [23]. In recent years, accumulated evidences have suggested BOLA2 was involved in HCC occurrence and development. While the expression and clinical relevance of BOLA gene family members in OC patients were still unclear.

Considering that many genes have been extensively selected as targets for cancer precision therapy, we explored the expression feature of the BOLA family members in $\mathrm{OC}$ using a series of bioinformatics methods to identify the potential targets for accurate therapy. Our data demonstrated that the expression of BOLA1, BOLA2 and BOLA3 in human OC tissues were all notably higher than normal ovarian tissues in Oncomine database, while BOLA2 and BOLA3 in human OC tissues were much higher than normal ovarian tissue in GEPIA database. Next, we found the protein expression of BOLA2 and BOLA3 were in human OC tissues were significantly higher than that in normal ovarian tissues in the HPA database, which was almost consistent with mRNA expression data of the Oncomine database and GEPIA database. Our data indicated that BOLA2 and BOLA3 may be the potential targets for accurate therapy for OC patients.

Just as everyone knows, many genes have been extensively used as predictive biomarkers to indicate the risk of recurrence of malignant tumors. Therefore, we explored the prognostic values of the BOLA family members in OC by using Kaplan-Meier plotter online database. Our data showed the increased BOLA3 mRNA level was correlated with shorter PFS and OS, while decreased BOLA2 mRNA level was associated with shorter PFS. In subgroup analysis, we found that abnormal mRNA expression of BOLA1 was closely related to prognosis of OC patients with histological grade G1-2, TP53 wild-type, and stage 3-4, abnormal mRNA expression of BOLA2 was closely related to prognosis of OC patients with serous pathological subtype, histological grade G1-3, TP53 wildtype and mutation, and stage 1-4, abnormal mRNA expression of BOLA3 was closely associated with prognosis of OC patients with serous pathological subtype, histological grade G1-3, and stage 3-4.these results indicated that indicated that BOLA1,BOLA2 and BOLA3 may be new prognostic biomarkers for patients with OC.

Next, to predict the potential mechanisms of BOLAs in the progression and prognosis of OC, we constructed the PPI network by using three BOLA members and 20 their interactors, and found BOLAs were associated with several metal-ion binding -related genes, including GLRX5, GLRX3, WRNIP1, ZNF576, THOP1, FAHD2A and HSCB. Previous studies showed GLRX3 [24], GLRX5 [25], WRNIP1 [26], THOP1 [27] and HSCB [28] were all regulators of various cancer occurrence and progression. Our data and present studies showed BOLA family members may mediate the progression and prognosis of OC by the interaction with these metal-ion binding-related genes. Then, we further investigate the alteration of BOLAs-related pathways in OC, and found BOLA1 was mainly related with the focal adhesion and oxidative phosphorylation in OC, BOLA3 was mainly related with oxidative phosphorylation, proteasome, protein export and glutathione metabolism in OC. Prevenient studies have reported the coordinated and dynamic regulation of focal adhesions is demanded for cell migration, which is much essential in cancer metastasis [29, 30].Other studies have indicated oxidative phosphorylation [31], proteasome [32], protein export [33] and glutathione metabolism [34] are all involved in cancer progression. Our data and previous studies showed BOLA family members may promote the progression and prognosis of $\mathrm{OC}$ by affecting these pathways, the precise mechanism needs clarify.

Finally, there are still some limitations in our study. Firstly, the clinical data available in these databases are finite, and data of several important factors including chemotherapy resistance, CA125 level, lymph node metastasis and tumor size that may effect on the prognosis of OC were missing. Secondly, the correlation between protein expression of BOLA family and OC prognosis is not clear. Thirdly, the precise mechanism of the BOLA family members' impact on OC patient prognosis has not been addressed. Finally, systematic tests on the expressions, roles and prognostic value of BOLA family have not been addressed.

In conclusion, the mRNA expressions, prognostic roles, genetic variations and PPI networks of BOLA family members in OC patients were investigated. This comprehensive bioinformatics analysis clarified that BOLA1, 2, and 3 may be optional prognostic biomarkers, and BOLA2 and BOLA3 may be a possible therapeutic target for precision therapy for patients with OC. Nevertheless, further experimental studies are urgently needed.

\section{Declarations}

\section{Availability of data and materials.}

Several publicly available datasets were used in this study and these data can be found in following websites: www.oncomine.org, http://gepia.cancerpku.cn/, http://kmplot.com/analysis/, https://www.cbioportal.org/, https://string-db.org/, https://david.ncifcrf.gov/.

\section{Authors' contributions}

All authors contributed to study design, data analysis, drafting, or revising the article, gave final approval of the version to be published, and agree to be accountable for all aspects of the work.

\section{Acknowledgements}

Not applicable.

\section{Funding}

Not applicable.

\section{Ethics approval and consent to participate}

Not applicable. 


\section{Patient consent for publication}

Not applicable.

\section{Competing interests}

The authors declare that they have no competing interests.

\section{References}

1. Siegel RL, Miller KD, Jemal A, Cancer statistics. 2018. CA: A Cancer Journal for Clinicians. 2018;68(1):7-30.

2. Bray F, Ferlay J, Soerjomataram I, Siegel RL, Torre LA, Jemal A. Global cancer statistics 2018: GLOBOCAN estimates of incidence and mortality worldwide for 36 cancers in 185 countries. CA Cancer J Clin. 2018;68(6):394-424.

3. Gunakan E, Tohma YA, Tunc M, Akilli H, Sahin H, Ayhan A. Factors associated with surgical morbidity of primary debulking in epithelial ovarian cancer. Obstet Gynecol Sci. 2020;63(1):64-71.

4. Matsuo K, Machida H, Yamagami W, et al. Intraoperative Capsule Rupture, Postoperative Chemotherapy, and Survival of Women With Stage I Epithelial Ovarian Cancer. Obstet Gynecol. 2019;134(5):1017-26.

5. Huynen MA, Spronk CA, Gabaldon T, Snel B. Combining data from genomes, $\mathrm{Y} 2 \mathrm{H}$ and $3 \mathrm{D}$ structure indicates that BolA is a reductase interacting with a glutaredoxin. FEBS Lett. 2005;579(3):591-6.

6. Li H, Outten CE. Monothiol CGFS glutaredoxins and BolA-like proteins: [2Fe-2S] binding partners in iron homeostasis. Biochemistry. 2012;51(22):437789.

7. Luo J, Wang D, Zhang S, et al. BolA family member 2 enhances cell proliferation and predicts a poor prognosis in hepatocellular carcinoma with tumor hemorrhage. J Cancer. 2019;10(18):4293-304.

8. Hunecke D, Spanel R, Langer F, Nam SW, Borlak J. MYC-regulated genes involved in liver cell dysplasia identified in a transgenic model of liver cancer. J Pathol. 2012;228(4):520-33.

9. Li H, Outten CE. Monothiol CGFS glutaredoxins and BolA-like proteins: [2Fe-2S] binding partners in iron homeostasis. Biochemistry. 2012;51(22):437789.

10. Tang Z, Li C, Kang B, Gao G, Li C, Zhang Z. GEPIA: a web server for cancer and normal gene expression profiling and interactive analyses. Nucleic Acids Res. 2017;45(W1):W98-102.

11. Lindskog C. The Human Protein Atlas - an important resource for basic and clinical research. Expert Rev Proteomics. 2016;13(7):627-9.

12. Gyorffy B, Lanczky A, Szallasi Z. Implementing an online tool for genome-wide validation of survival-associated biomarkers in ovarian-cancer using microarray data from 1287 patients. Endocr Relat Cancer. 2012;19(2):197-208.

13. Gyorffy B, Lanczky A, Eklund AC, et al. An online survival analysis tool to rapidly assess the effect of 22,277 genes on breast cancer prognosis using microarray data of 1,809 patients. Breast Cancer Res Treat. 2010;123(3):725-31.

14. Gyorffy B, Surowiak P, Budczies J, Lanczky A. Online survival analysis software to assess the prognostic value of biomarkers using transcriptomic data in non-small-cell lung cancer. PLoS One. 2013;8(12):e82241.

15. Győrffy B, Surowiak P, Budczies J, Lánczky A. Online survival analysis software to assess the prognostic value of biomarkers using transcriptomic data in non-small-cell lung cancer. PLoS One. 2013;8(12):e82241.

16. Gao J, Aksoy BA, Dogrusoz U, et al. Integrative analysis of complex cancer genomics and clinical profiles using the cBioPortal. Sci Signal. 2013;6(269):pl1.

17. Warde-Farley D, Donaldson SL, Comes O, et al. The GeneMANIA prediction server: biological network integration for gene prioritization and predicting gene function. Nucleic Acids Res. 2010;38(Web Server issue):W214-20.

18. Szklarczyk D, Morris JH, Cook H, et al. The STRING database in 2017: quality-controlled protein-protein association networks, made broadly accessible. Nucleic Acids Res. 2017;45(D1):D362-D8.

19. Su G, Morris JH, Demchak B, Bader GD. Biological network exploration with Cytoscape 3. Curr Protoc Bioinformatics. 2014;47:8.13.1-24..

20. Huang da W, Sherman BT, Lempicki RA. Systematic and integrative analysis of large gene lists using DAVID bioinformatics resources. Nat Protoc. 2009;4(1):44-57.

21. Santos I, Ramos C, Mendes C, Sequeira CO, et al. Targeting Glutathione and Cystathionine $\beta$-Synthase in Ovarian Cancer Treatment by SeleniumChrysin Polyurea Dendrimer Nanoformulation. Nutrients. 2019;11(10):2523.

22. Frey AG, Palenchar DJ, Wildemann JD. Philpott CC. A Glutaredoxin.BolA Complex Serves as an Iron-Sulfur Cluster Chaperone for the Cytosolic Cluster Assembly Machinery. J Biol Chem. 2016;291(43):22344-56.

23. Bogdan AR, Miyazawa M, Hashimoto K, Tsuji Y. Regulators of Iron Homeostasis: New Players in Metabolism, Cell Death, and Disease. Trends Biochem Sci. 2016;41(3):274-86.

24. Li B, Chen M, Lu M, Xin-Xiang J, Meng-Xiong P, Jun-Wu M. Glutaredoxin 3 promotes migration and invasion via the Notch signalling pathway in oral squamous cell carcinoma. Free Radic Res. 2018;52(4):390-401. 
25. Lee J, You JH, Shin D, Roh JL. Inhibition of Glutaredoxin 5 predisposes Cisplatin-resistant Head and Neck Cancer Cells to Ferroptosis. Theranostics. 2020;10(17):7775-86.

26. Jiang W, Han X, Wang J, et al. miR-22 enhances the radiosensitivity of small-cell lung cancer by targeting the WRNIP1. J Cell Biochem. 2019;120(10):17650-61.

27. Qi L, Li SH, Si LB, Lu M, Tian H. Expression of THOP1 and its relationship to prognosis in non-small cell lung cancer. PLoS One. 2014;9(9):e106665.

28. Tong WH, Sourbier C, Kovtunovych G, et al. The glycolytic shift in fumarate-hydratase-deficient kidney cancer lowers AMPK levels, increases anabolic propensities and lowers cellular iron levels. Cancer Cell. 2011;20(3):315-27.

29. Huang HK, Lin YH, Chang HA, et al. Chemoresistant ovarian cancer enhances its migration abilities by increasing store-operated Ca(2+) entry-mediated turnover of focal adhesions. J Biomed Sci. 2020;27(1):36.

30. Murphy JM, Rodriguez YAR, Jeong K, Ahn EE, Lim SS. Targeting focal adhesion kinase in cancer cells and the tumor microenvironment. Exp Mol Med. 2020;52(6):877-86.

31. Ashton TM, McKenna WG, Kunz-Schughart LA, Higgins GS. Oxidative Phosphorylation as an Emerging Target in Cancer Therapy. Clin Cancer Res. 2018;24(11):2482-90.

32. Mofers A, Pellegrini P, Linder S, D'Arcy P. Proteasome-associated deubiquitinases and cancer. Cancer Metastasis Rev. 2017;36(4):635-53.

33. Nachmias B, Schimmer AD. Targeting nuclear import and export in hematological malignancies. Leukemia. 2020.

34. Santos I, Ramos C, Mendes C, et al. Targeting Glutathione and Cystathionine beta-Synthase in Ovarian Cancer Treatment by Selenium-Chrysin Polyurea Dendrimer Nanoformulation. Nutrients. 2019;11(10).

\section{Tables}

Table I. The significant changes of BOLA family members expression between different types of OC and normal tissues (Oncomine)

\begin{tabular}{|c|c|c|c|c|c|c|c|c|c|c|c|c|c|c|c|c|}
\hline \multirow[t]{3}{*}{ BOLA } & \multicolumn{15}{|c|}{ Types of OC vs. Normal } & \multirow[t]{3}{*}{ Ref/Source } \\
\hline & \multicolumn{3}{|c|}{ Ovarian Carcinoma } & \multicolumn{3}{|c|}{ Endometrioid } & \multicolumn{3}{|c|}{ Clear cell } & \multicolumn{3}{|c|}{ Serous } & \multicolumn{3}{|c|}{ Mucinous } & \\
\hline & $\mathrm{FC}$ & $\mathbf{P}$ & $\mathbf{N}$ & FC & $\mathbf{P}$ & $\mathbf{N}$ & FC & $\mathbf{P}$ & $\mathbf{N}$ & FC & $\mathbf{P}$ & $\mathbf{N}$ & FC & $\mathbf{P}$ & $\mathbf{N}$ & \\
\hline \multirow[t]{3}{*}{ BOLA1 } & 1.373 & $<0.001$ & 195 & - & - & - & - & - & - & - & - & - & - & - & - & Bonomeovarian \\
\hline & - & - & - & 1.268 & $<0.001$ & 9 & 1.191 & 0.004 & 7 & 1.180 & 0.001 & 20 & 1.096 & 0.027 & 9 & Lu ovarian \\
\hline & - & - & - & 1.032 & 0.709 & 37 & 1.093 & 0.912 & 8 & 1.005 & 0.468 & 41 & 1.072 & 0.867 & 13 & Hendrix ovarian \\
\hline \multirow[t]{3}{*}{ BOLA2 } & 3.545 & $<0.001$ & 195 & - & - & - & - & - & - & - & - & - & - & - & - & $\begin{array}{l}\text { Bonome } \\
\text { ovarian }\end{array}$ \\
\hline & - & - & - & 2.098 & $<0.001$ & 9 & 2.083 & $<0.001$ & 7 & 2.166 & $<0.001$ & 20 & - & - & - & Lu ovarian \\
\hline & - & - & - & 1.196 & 0.003 & 37 & 1.228 & 0.002 & 8 & 1.191 & 0.003 & 41 & 1.191 & 0.005 & 13 & Hendrix ovarian \\
\hline BOLA3 & - & - & - & 2.003 & 0.001 & 9 & 1.455 & 0.065 & 7 & 1.537 & $<0.001$ & 20 & 1.490 & 0.024 & 9 & Lu ovarian \\
\hline
\end{tabular}

The bold font indicates the difference between $\mathrm{OC}$ and normal tissues conformedtothe selected thresholds. OC, ovarian cancer;

FC, fold change; "-", not available; N, number of patients.

Table II. The relationship between BOLA family members and OS in other different subtypes of OC (Kaplan-Meier plotter) 


\begin{tabular}{|c|c|c|c|c|c|c|c|c|c|c|}
\hline \multirow[t]{2}{*}{ Subtypes } & & \multicolumn{3}{|l|}{ BOLA1 } & \multicolumn{3}{|l|}{ BOLA2 } & \multicolumn{3}{|l|}{ BOLA3 } \\
\hline & & Cases & $\mathrm{HR}(95 \% \mathrm{Cl})$ & P value & Cases & $\mathrm{HR}(95 \% \mathrm{Cl})$ & P value & Cases & $\mathrm{HR}(95 \% \mathrm{Cl})$ & Pvalue \\
\hline \multirow[t]{2}{*}{ Histology } & Serous & 1207 & $0.93(0.8-1.08)$ & 0.34 & 1207 & $0.86(0.73-1.01)$ & 0.061 & 523 & $1.36(1.04-1.77)$ & 0.022 \\
\hline & Endometrioid & 37 & $2.13(0.24-19.05)$ & 0.49 & 37 & 4.42(0.74-26.51) & 0.075 & 30 & $3.77(0.39-36.42)$ & 0.22 \\
\hline \multirow[t]{4}{*}{ Stage } & 1 & 74 & $2.29(0.73-7.25)$ & 0.15 & 74 & $0.43(0.14-1.35)$ & 0.14 & 51 & $2.52(0.6-10.55)$ & 0.19 \\
\hline & 2 & 61 & $0.52(0.18-1.5)$ & 0.22 & 61 & $0.28(0.08-1.01)$ & 0.038 & 32 & $0.16(0.02-1.36)$ & 0.057 \\
\hline & 3 & 1044 & $1.15(0.97-1.36)$ & 0.097 & 1044 & $1.15(0.95-1.38)$ & 0.15 & 426 & $1.38(1.05-1.81)$ & 0.019 \\
\hline & 4 & 176 & $1.38(0.95-2.01)$ & 0.087 & 176 & $0.69(0.47-1.02)$ & 0.059 & 61 & $0.49(0.26-0.9)$ & 0.019 \\
\hline \multirow[t]{2}{*}{ Grade } & $1+2$ & 380 & $1.54(1.16-2.06)$ & 0.003 & 380 & $0.79(0.6-1.06)$ & 0.11 & 203 & $1.54(0.97-2.46)$ & 0.064 \\
\hline & 3 & 1015 & $1.11(0.92-1.34)$ & 0.28 & 1015 & $0.82(0.7-0.98)$ & 0.025 & 392 & $0.73(0.56-0.96)$ & 0.022 \\
\hline \multirow[t]{2}{*}{ TP53 } & Mutated & 506 & $0.84(0.65-1.08)$ & 0.17 & 506 & $0.71(0.56-0.89)$ & 0.0025 & 124 & $0.79(0.54-1.16)$ & 0.23 \\
\hline & WT & 94 & $0.5(0.29-0.89)$ & 0.015 & 94 & $0.54(0.25-1.14)$ & 0.1 & 19 & $1.38(0.49-3.92)$ & 0.54 \\
\hline \multirow[t]{3}{*}{$\mathrm{CR}$} & Platin & 1409 & $1.14(0.99-1.32)$ & 0.07 & 1409 & $0.94(0.81-1.09)$ & 0.41 & 478 & $1.30(1-1.69)$ & 0.047 \\
\hline & Taxol & 793 & $1.16(0.95-1.4)$ & 0.14 & 793 & $1.17(0.97-1.41)$ & 0.11 & 357 & $1.39(1.01-1.93)$ & 0.043 \\
\hline & Taxol + platin & 776 & $1.17(0.97-1.43)$ & 0.10 & 776 & $1.19(0.98-1.44)$ & 0.075 & 356 & $1.40(1.01-1.93)$ & 0.041 \\
\hline
\end{tabular}

The bold font indicates the difference was significant statistically. OC, ovarian cancer; OS, overall survival; WT, wild type; CR, Chemotherapy Regimen

Table III. The relationship between BOLA family members and PFS in other different subtypes of OC (Kaplan-Meier plotter)

\begin{tabular}{|c|c|c|c|c|c|c|c|c|c|c|}
\hline \multirow[t]{2}{*}{ Subtypes } & & \multicolumn{3}{|l|}{ BOLA1 } & \multicolumn{3}{|l|}{ BOLA2 } & \multicolumn{3}{|l|}{ BOLA3 } \\
\hline & & Cases & $\mathrm{HR}(95 \% \mathrm{Cl})$ & P value & Cases & $\mathrm{HR}(95 \% \mathrm{Cl})$ & $P$ value & Cases & $\mathrm{HR}(95 \% \mathrm{Cl})$ & Pvalue \\
\hline \multirow[t]{2}{*}{ Histology } & Serous & 483 & $0.93(0.81-1.08)$ & 0.33 & 483 & $0.8(0.69-0.93)$ & 0.0048 & 483 & $1.36(1.08-1.72)$ & 0.0091 \\
\hline & Endometrioid & 44 & $2.19(0.84-5.7)$ & 0.10 & 44 & $2.05(0.81-5.21)$ & 0.12 & 44 & $1.95(0.44-8.72)$ & 0.37 \\
\hline \multirow[t]{4}{*}{ Stage } & 1 & 96 & $1.72(0.59-5.01)$ & 0.32 & 96 & $0.27(0.1-0.79)$ & 0.01 & 74 & $0.62(0.17-2.2)$ & 0.46 \\
\hline & 2 & 67 & $1.6(0.69-3.73)$ & 0.27 & 67 & $0.29(0.12-0.72)$ & 0.0047 & 41 & $1.94(0.56-6.73)$ & 0.29 \\
\hline & 3 & 919 & $0.82(0.71-0.96)$ & 0.013 & 919 & $0.81(0.7-0.95)$ & 0.0083 & 424 & $1.21(0.95-1.53)$ & 0.13 \\
\hline & 4 & 162 & $1.52(1.04-2.24)$ & 0.031 & 162 & $0.59(0.38-0.92)$ & 0.018 & 70 & $0.60(0.34-1.07)$ & 0.082 \\
\hline \multirow[t]{2}{*}{ Grade } & $1+2$ & 293 & $1.48(1.04-2.1)$ & 0.028 & 293 & $0.69(0.52-0.92)$ & 0.0098 & 189 & $1.66(1.13-2.44)$ & 0.0085 \\
\hline & 3 & 837 & $1.11(0.92-1.34)$ & 0.3 & 837 & $0.85(0.72-1)$ & 0.049 & 315 & $0.74(0.55-0.99)$ & 0.04 \\
\hline \multirow[t]{2}{*}{ TP53 } & Mutated & 483 & $1.24(0.99-1.56)$ & 0.065 & 483 & $0.7(0.56-0.88)$ & 0.0018 & 124 & $1.30(0.87-1.94)$ & 0.19 \\
\hline & WT & 84 & $0.62(0.36-1.06)$ & 0.077 & 84 & $0.54(0.31-0.95)$ & 0.031 & 19 & $0.60(0.21-1.73)$ & 0.34 \\
\hline \multirow[t]{3}{*}{ CR } & Platin & 1259 & $0.85(0.75-0.97)$ & 0.018 & 1259 & $0.79(0.69-0.91)$ & $<0.001$ & 502 & $0.81(0.65-1)$ & 0.054 \\
\hline & Taxol & 715 & $0.87(0.74-1.04)$ & 0.12 & 715 & $0.8(0.67-0.95)$ & 0.011 & 381 & $1.30(1-1.69)$ & 0.046 \\
\hline & Taxol + platin & 698 & $0.89(0.75-1.06)$ & 0.2 & 698 & $0.81(0.68-0.97)$ & 0.021 & 380 & 1.301-1.69) & 0.049 \\
\hline
\end{tabular}

The bold font indicates the difference was significant statistically. OC, ovarian cancer; PFS, progression-free survival; WT, wild type; CR, Chemotherapy Regimen

Figures 


\section{BOLAl BOLA2 BOLA3}

\begin{tabular}{|c|c|c|c|c|}
\hline \multirow{2}{*}{$\begin{array}{l}\text { Analysis Type by Cancer } \\
\text { Bladder Cancer }\end{array}$} & $\begin{array}{c}\begin{array}{c}\text { Cancer } \\
\text { vs. } \\
\text { Normal }\end{array}\end{array}$ & $\begin{array}{c}\text { Cancer } \\
\text { vs. } \\
\text { Normal }\end{array}$ & \multicolumn{2}{|c|}{$\begin{array}{c}\begin{array}{c}\text { Cancer } \\
\text { vs. } \\
\text { Normal }\end{array}\end{array}$} \\
\hline & 1 & $\frac{3}{3}$ & & \\
\hline Brain and CNS Cancer & 1 & 2 & & 1 \\
\hline Breast Cancer & 2 & 16 & 7 & 1 \\
\hline Cenvical Cancer & & 1 & & \\
\hline Colorectal Cancer & & 1 & 1 & \\
\hline Esophageal Cancer & 1 & 1 & 1 & \\
\hline Gastric Cancer & & & & \\
\hline Head and Neck Cancer & & 2 & 1 & \\
\hline Kidney Cancer & & 2 & 2 & \\
\hline Leukemia & & 1 & & 4 \\
\hline Liver Cancer & 2 & 3 & & \\
\hline Lung Cancer & 1 & 13 & 3 & \\
\hline Lymphoma & 1 & 9 & 7 & \\
\hline Melanoma & 1 & & & \\
\hline Myeloma & & 2 & & \\
\hline Other Cancer & & 1 & & \\
\hline Ovarian Cancer & & 6 & 1 & \\
\hline Pancreatic Cancer & & 1 & & \\
\hline Prostate Cancer & & 3 & & \\
\hline Sarcoma & & 1 & & \\
\hline Significant Unique Analyses & 101 & \begin{tabular}{l|l}
66 & 5 \\
\end{tabular} & 23 & 6 \\
\hline Total Unique Analyses & 289 & 314 & 179 & 79 \\
\hline
\end{tabular}

\section{$\stackrel{1}{\square} \stackrel{10}{\square}^{10} \stackrel{10}{\square}^{5} \stackrel{1}{\square}^{1}$}

Cell color is determined by the best gene rank percentile for the analyses within the cell.

NOTE: An analysis may be counted in more than one cancer type.

\section{Figure 1}

Expression levels of BOLA family members in different types of cancers (Oncomine). The number of each cell represents the number of analyses that conformed to the following threshold: $\mathrm{P}<0.001$, the absolute value of fold change $>1.5$, and gene rank, $10 \%$. The numbers in colored cells show the quantities of datasets with statistically significant mRNA higher expression (red) or lower expression (blue) of BOLA family members. 
A
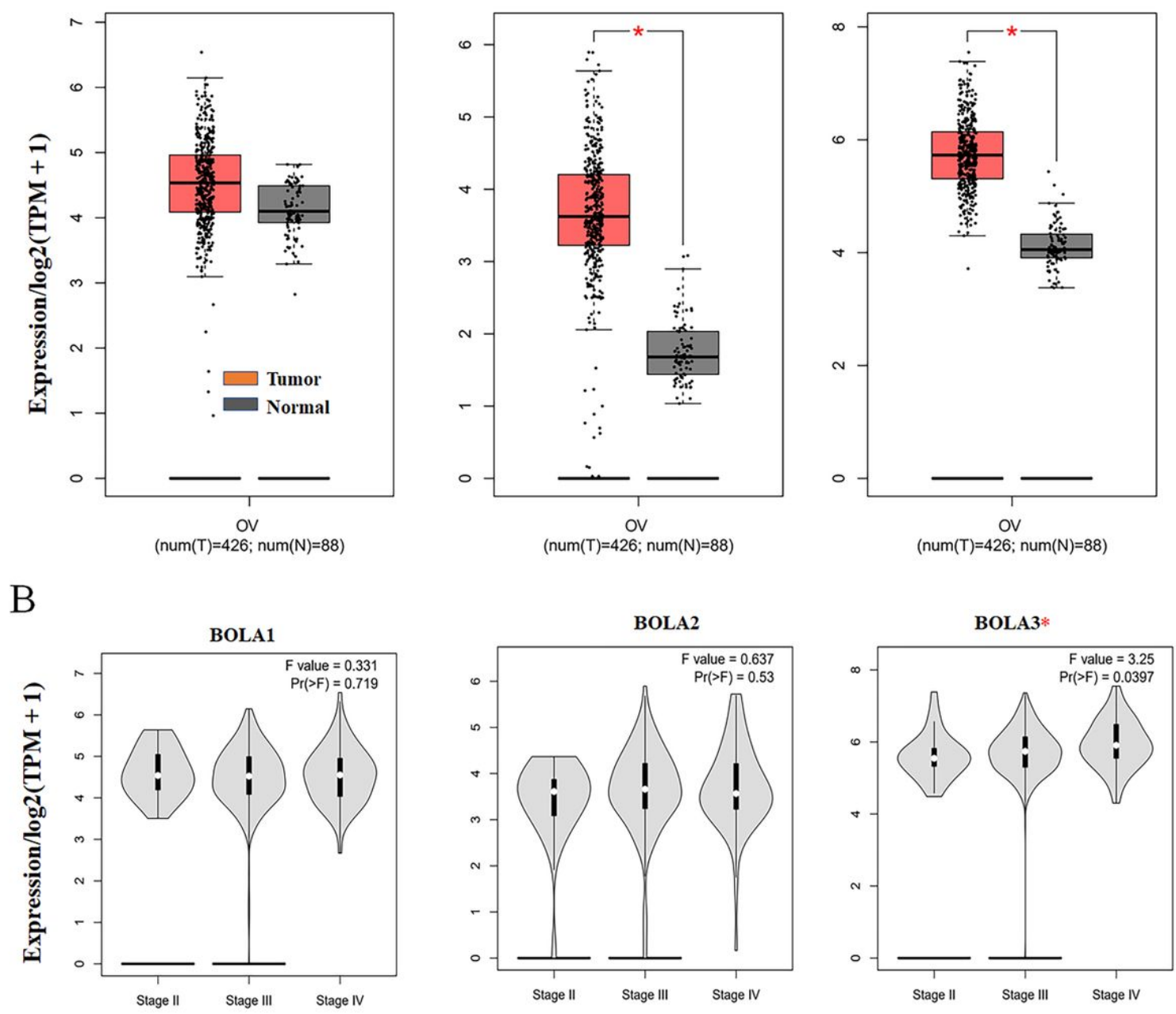

\section{Figure 2}

Dysregulated mRNA expression levels of BOLA family members and the association with tumor stage in OC patients. (A) Box plots of dysregulated mRNA expression BOLA family members in OC based on GEPIA database. (B) The relationship between mRNA expression of BOLA family members and tumor stages in $\mathrm{OC}$ patients. 
A

BOLA1

BOLA2

BOLA3

Normal

Tumor
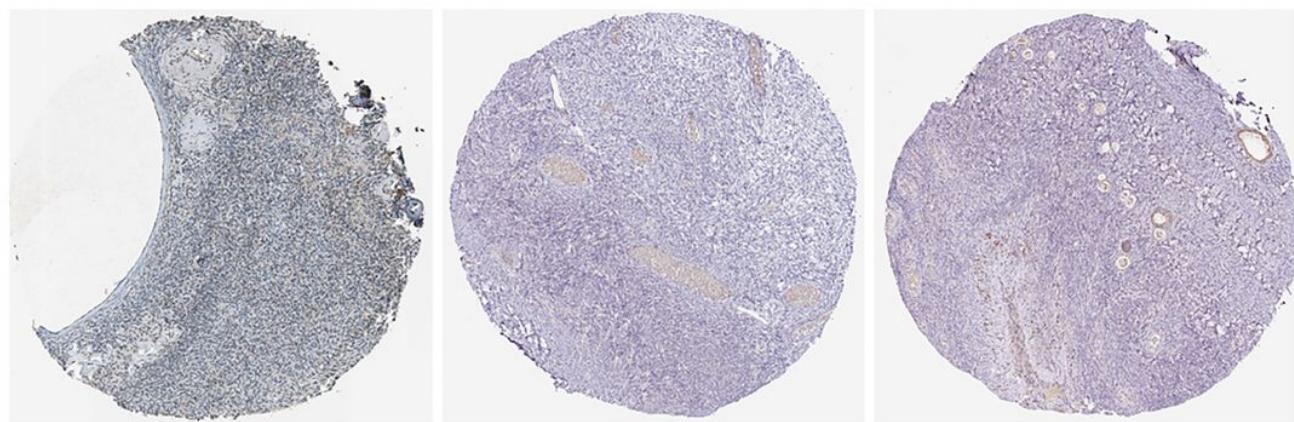

$4 x^{2}$.
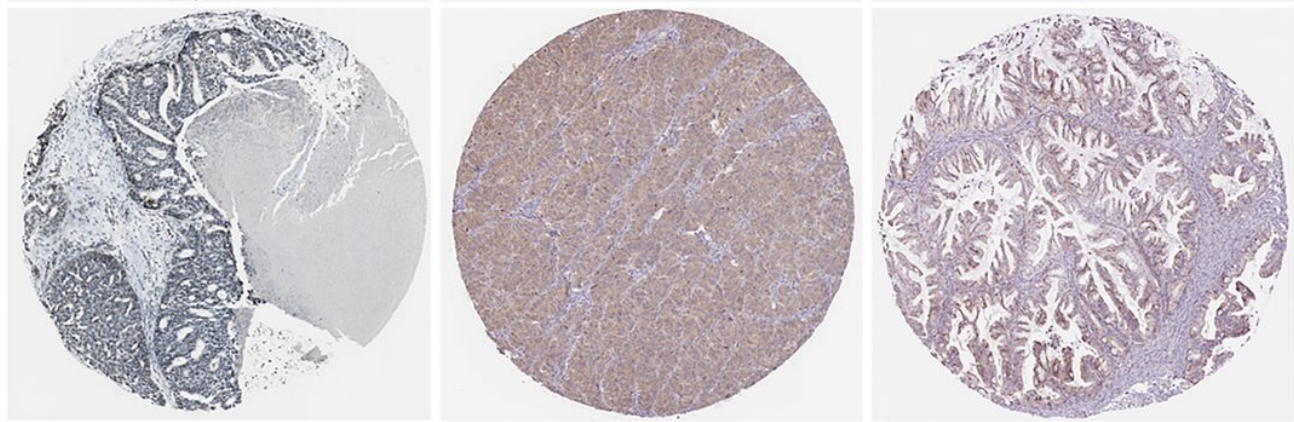

B
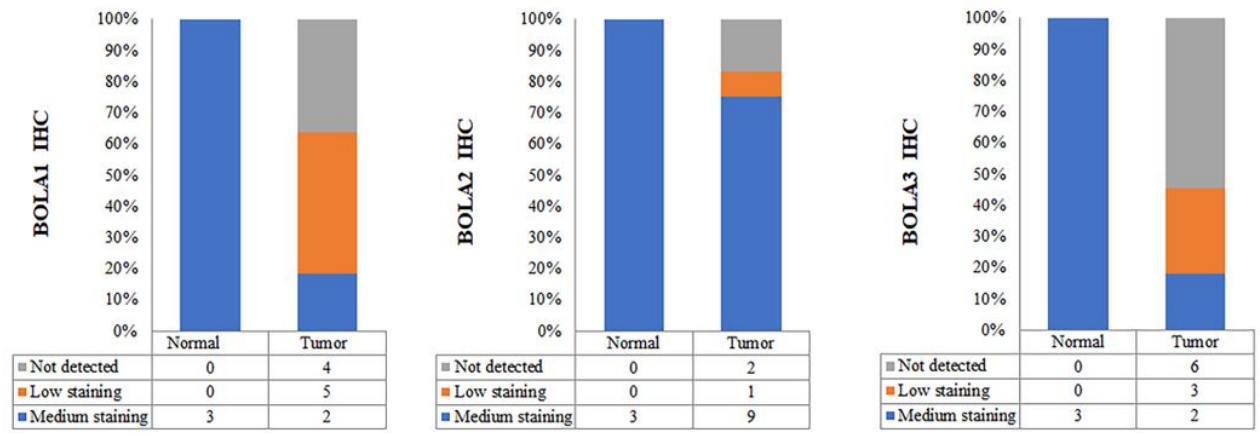

Figure 3

Comparison of the protein expression profile of BOLA family members in normal ovarian tissues and ovarian cancer tissues based on HPA database. Representative immunohistochemistry images (A) and expression status (B) of BOLA family members in normal ovarian tissues and ovarian cancer tissues based on HPA database (http://www.proteinatlas.org/). 
A

PFS

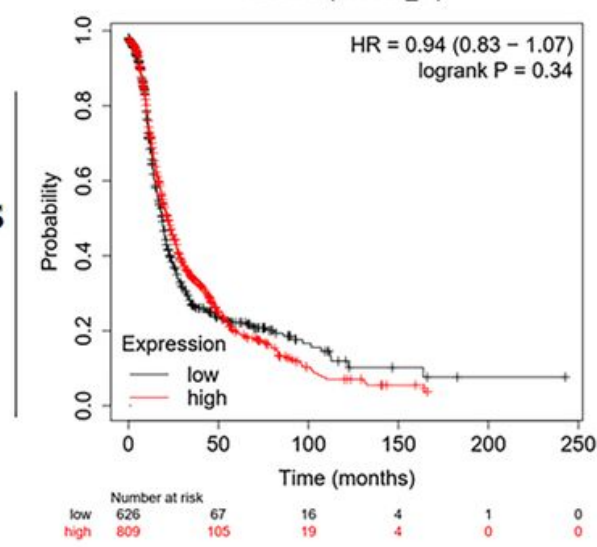

D

BOLA1 (219345_at)

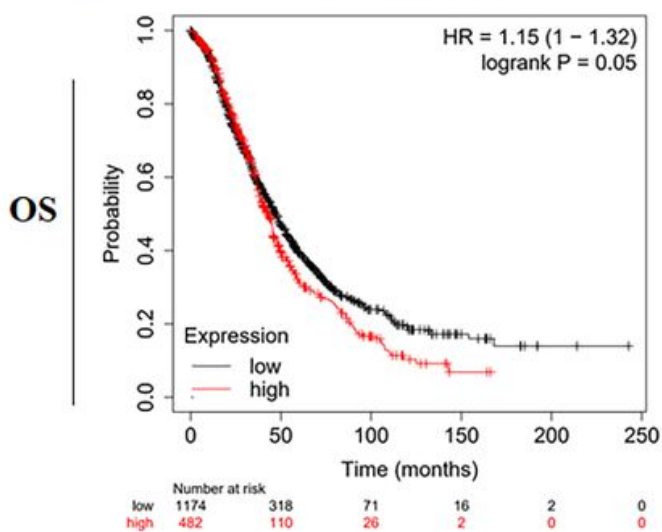

B

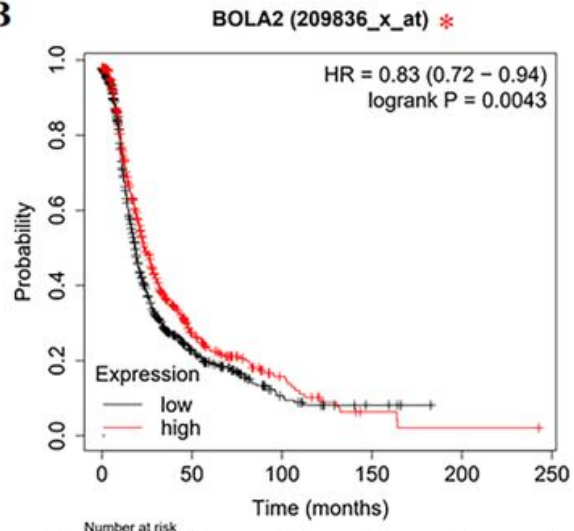

E

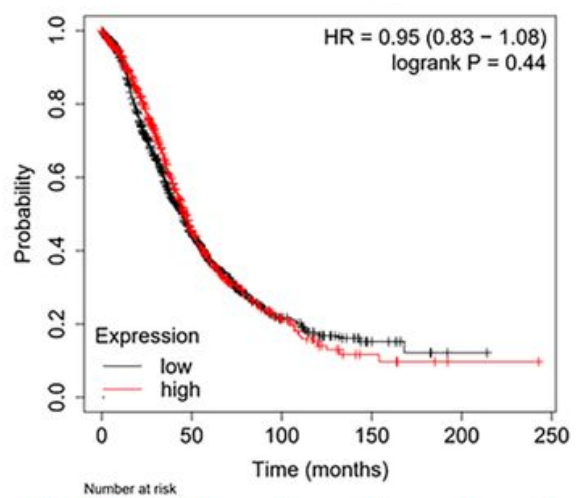

C

BOLA3 (227291_s_at) *

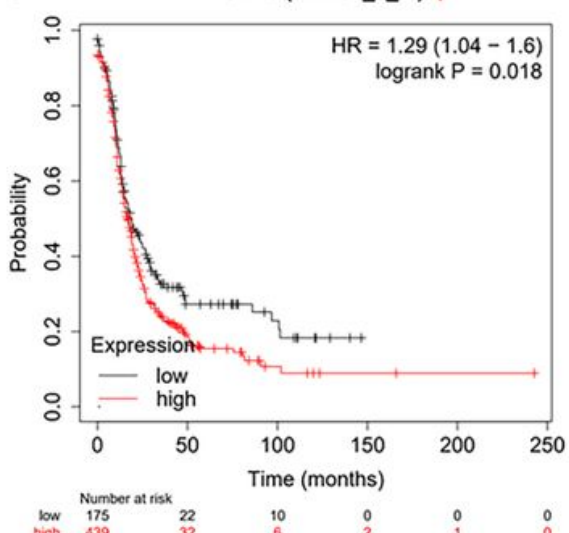

F

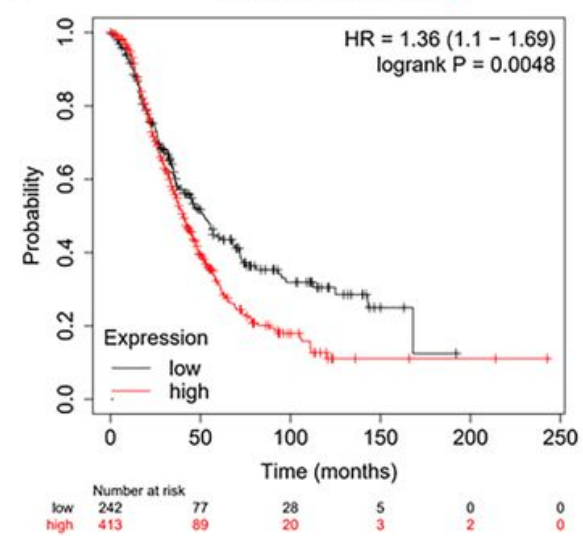

Figure 4

Prognostic values of mRNA level of BOLA family members in OC patients based on Kaplan-Meier plotter database. Kaplan-Meier curves show the correlation between mRNA expression of BOLA family members and progression-free survival (A-C), and overall survival (D-F) of $O C$ patients. ${ }^{*} P<0.05$. 
A
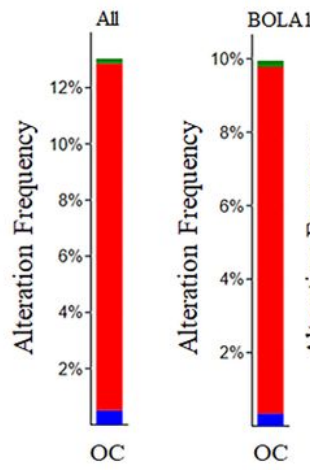

$\mathrm{C}$

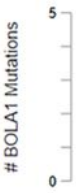

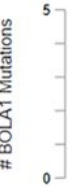

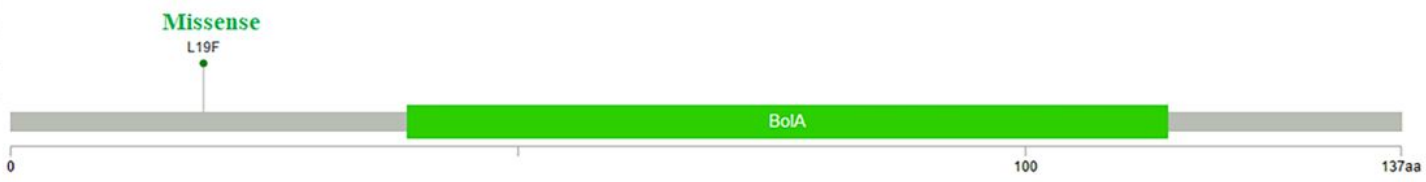

$\mathrm{D}$
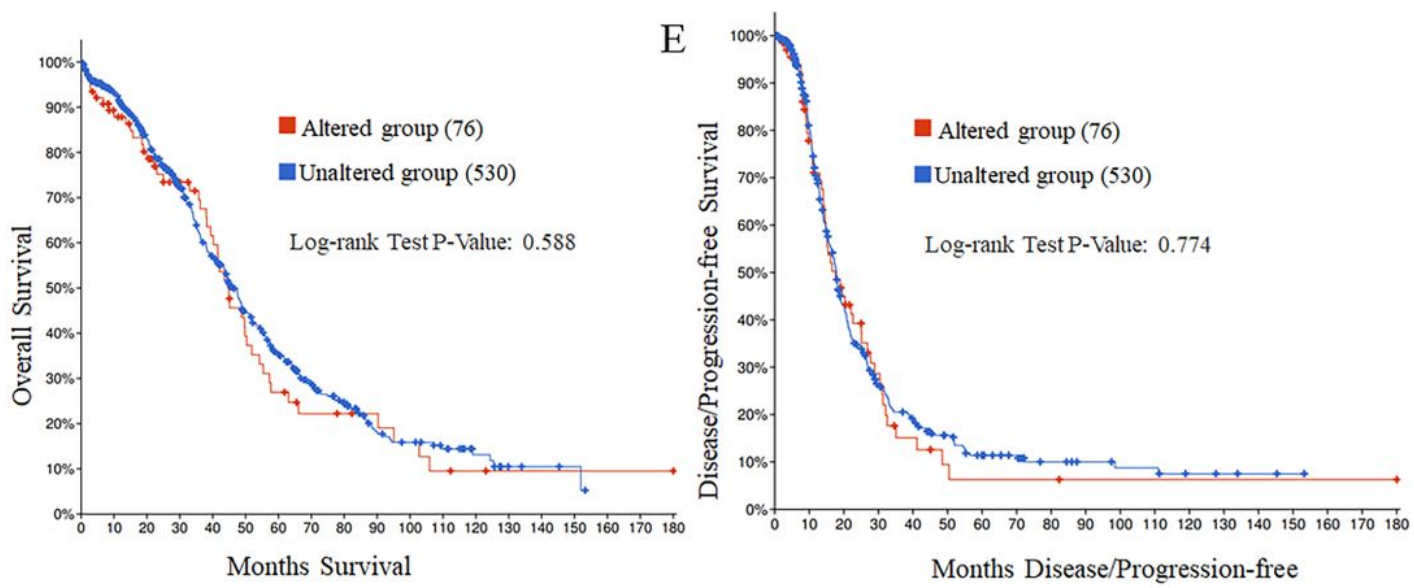

\section{Figure 5}

Genomic analysis of BOLA family members in OC based on cBioPortal databses. (A) Summary of genomic alteration of BOLA family members in OC. (B) Oncoprint visual summary of alteration on a query of BOLA family members in OC. (C) The mutations of BOLA1 was plotted. Kaplan-Meier plots comparing (D) overall survival (OS) and (E) progression-free survival (PFS) in cases with/without BOLA family members' gene alterations. 
A

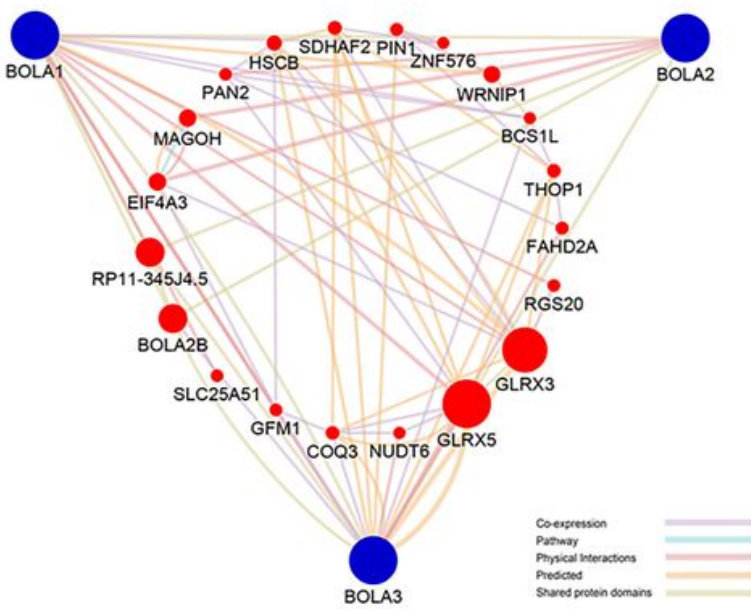

B

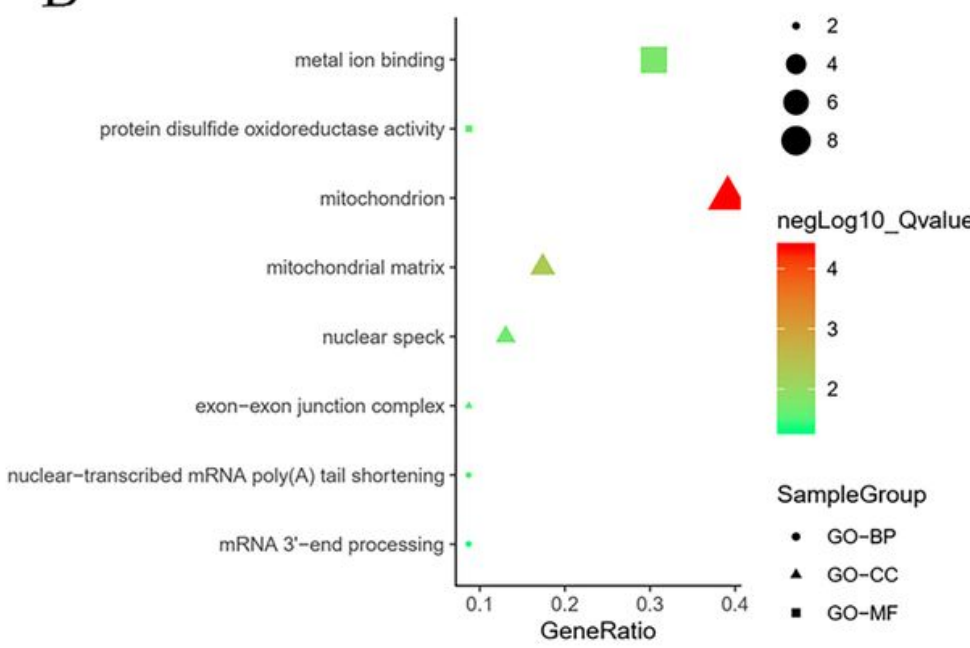

$\mathrm{C}$

\begin{tabular}{|c|c|c|c|c|c|c|c|}
\hline & BOLA1 & BOLA2 & BOLA3 & & & \multirow{8}{*}{$\begin{array}{l}\text { BOLA1 } \\
\text { BOLA2 } \\
\text { BOLA3 }\end{array}$} \\
\hline BOLA1 & 1 & 0.24 & 0.21 & \multirow{3}{*}{\multicolumn{3}{|c|}{ cBioPortal }} & \\
\hline BOLA2 & 0.24 & 1 & 0.32 & & & & \\
\hline BOLA3 & 0.21 & 0.32 & 1 & & & & \\
\hline & \multirow{4}{*}{\multicolumn{2}{|c|}{ GEPIA }} & & 1 & 0.52 & 0.43 & \\
\hline & & & & 0.52 & 1 & 0.56 & \\
\hline & & & & 0.43 & 0.56 & 1 & \\
\hline & & & & BOLA1 & BOLA2 & BOLA3 & \\
\hline
\end{tabular}

Figure 6

Protein-protein interaction (PPI) and function enrichment of BOLA family members. (A) The network of 3 BOLA family members and 20 proteins that significantly interacted with BOLA family members (String). (B) Gene Ontology (GO) enrichment analysis of BOLA family members and their interactors (DAVID). (C) The Pearson correlation coefficients between BOLA family members based on GEPIA and cBioPortal databases. 
A

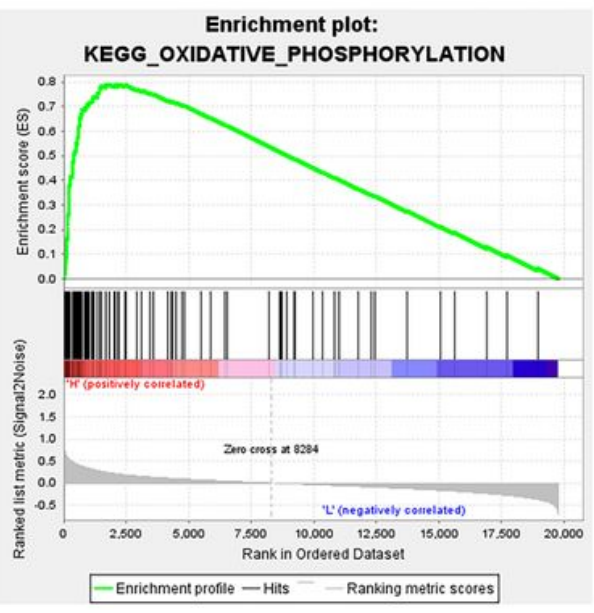

D

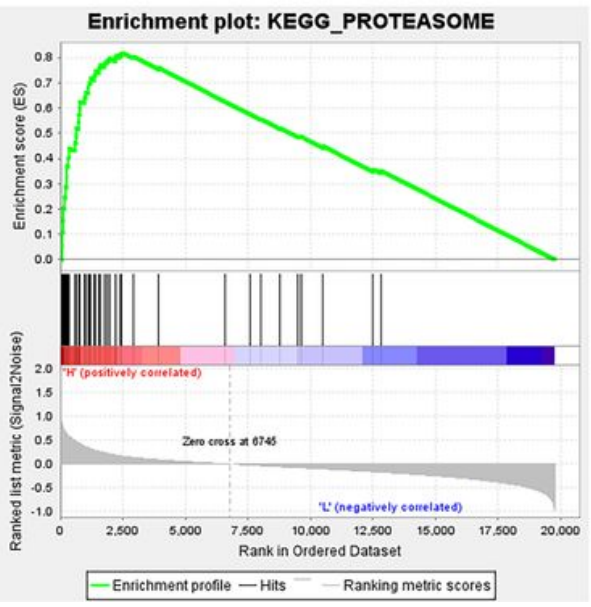

B

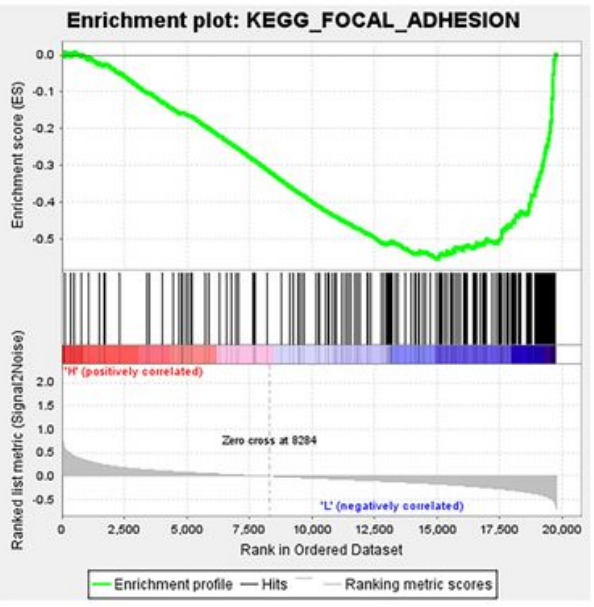

E

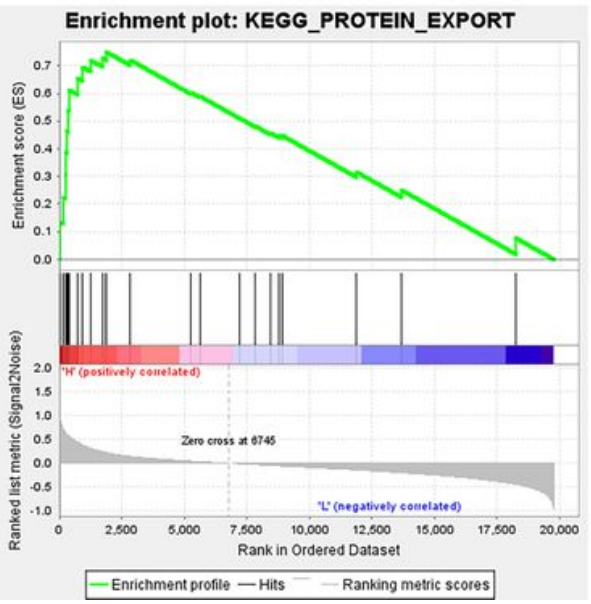

C

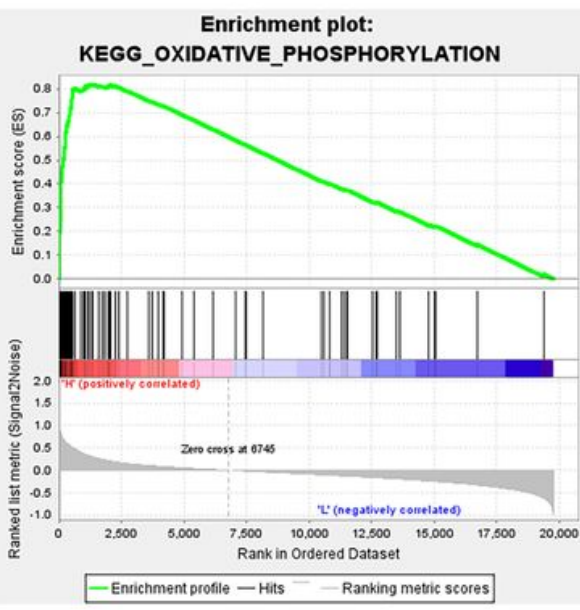

F

Enrichment plot: KEGG_GLUTATHIONE_METABOLISM

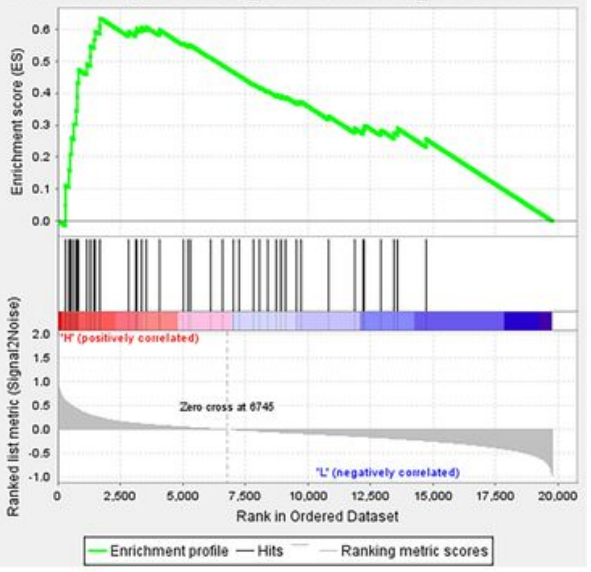

Figure 7

Gene set enrichment analysis (GSEA) analysis of BOLA family members. (A) GSEA showed that BOLA1 expression was positively correlated with oxidative phosphorylation. (B) GSEA showed that BOLA1 expression was negatively correlated with the focal adhesion. GSEA showed that BOLA3 expression was positively correlated with oxidative phosphorylation (C), proteasome (D), proteins export (E), and glutathione metabolism (F) in OC. 